\title{
No New Cosmological Concordance with Massive Sterile Neutrinos
}

\author{
Boris Leistedt, ${ }^{1, *}$ Hiranya V. Peiris, ${ }^{1, \dagger}$ and Licia Verde ${ }^{2,3, *}$ \\ ${ }^{1}$ Department of Physics and Astronomy, University College London, London WC1E 6BT, United Kingdom \\ ${ }^{2}$ ICREA (Institució Catalana de Recerca i Estudis Avançat) and ICC, Institut de Ciencies del Cosmos, \\ Universitat de Barcelona (UB-IEEC), Marti i Franques 1, Barcelona 08028, Spain \\ ${ }^{3}$ Institute of Theoretical Astrophysics, University of Oslo, 0315 Oslo, Norway
}

(Received 9 May 2014; revised manuscript received 25 June 2014; published 21 July 2014)

\begin{abstract}
It has been claimed recently that massive sterile neutrinos could bring about a new concordance between observations of the cosmic microwave background, the large-scale structure of the Universe, and local measurements of the Hubble constant, $H_{0}$. We demonstrate that this apparent concordance results from combining data sets which are in significant tension, even within this extended model, possibly indicating remaining systematic biases in the measurements. We further show that this tension remains when the cosmological model is further extended to include significant tensor modes, as suggested by the recent BICEP2 results. Using the Bayesian evidence, we show that the cold dark matter model with a cosmological constant is strongly favored over its neutrino extensions by various combinations of data sets. Robust data combinations yield stringent limits of $\sum m_{\nu} \lesssim 0.3 \mathrm{eV}$ and $m_{\nu, \text { sterile }}^{\text {eff }} \lesssim 0.3 \mathrm{eV}$ at $95 \%$ C.L. for the sum of active and sterile neutrinos, respectively.
\end{abstract}

DOI: 10.1103/PhysRevLett.113.041301

PACS numbers: 98.80.Es, 14.60.St, 98.70.Vc

The temperature fluctuations of the cosmic microwave background (CMB), as measured by the Planck satellite [1], have yielded subpercent level constraints on the cosmological parameters of the vanilla $\Lambda \mathrm{CDM}$ model. However, the primary $\mathrm{CMB}$ temperature fluctuations only indirectly probe the growth of cosmic structure, and it is therefore essential to complement it with observations large-scale structure (LSS) such as galaxy clusters, weak lensing, and clustering measurements. The first cosmological results from the Planck satellite have revealed a $\sim 2 \sigma$ tension between CMB temperature measurements and the Sunyaev-Zel'dovich (SZ) cluster abundances [2], mainly in terms of $\sigma_{8}$, the linear-theory mass dispersion on a scale of $8 h^{-1} \mathrm{Mpc}$. A similar tension is observed with the x-ray cluster counts [3].

Massive neutrinos can potentially alleviate this tension because they suppress power in the clustering of matter at late times. They are an appealing solution since solar and atmospheric experiments have already provided evidence for their mass, with room for extra sterile species, supported by anomalies in short baseline and reactor neutrino experiments (for reviews of particle physics constraints see, e.g., Refs. [4-7]). Cluster abundances, galaxy surveys and weak lensing are sensitive to the total neutrino mass, either from active neutrinos $\sum m_{\nu}$ (the total mass from active species), or sterile neutrinos $m_{\nu, \text { sterile }}^{\text {eff }}$ (an effective parameter that connects to actual neutrino masses in the context of specific models-see, e.g., Ref. [8]). In addition, an extra parameter $\mathrm{N}_{\text {eff }}$ can be introduced to denote the effective number of relativistic species, in which case $\mathrm{N}_{\text {eff }}>3.046$ (the standard number) is referred to as "dark radiation" and is also appealing as it could alleviate the tension between Planck and local $H_{0}$ measurements [9].
A number of recent studies have carried out joint analyses of various data combinations to conclude that these tensions are resolved within a new concordance model which implies nonstandard neutrino parameters [10-14]. Reference [10] argued that combining the CMB with lensing or SZ cluster measurements reveals evidence for nonzero neutrino mass in both the active and sterile neutrino scenarios. References [11,12] claimed that sterile neutrinos could reconcile Planck with LSS data, in particular with the x-ray cluster abundances [3] and the latest constraints on $H_{0}$ [15]. By combining the CMB with shear and redshift space distortion (RSD) measurements, Ref. [13] found hints of nonzero masses for active neutrinos. Finally, Refs. [16,17] further claimed that sterile neutrinos could resolve a potential tension between Planck and BICEP [18] constraints on $r_{0.002}$, the tensor-to-scalar ratio at $k=0.002 \mathrm{Mpc}^{-1}$.

Although these conclusions are not universally accepted [1,19-22], tension between the data sets may indeed point to new physics. Alternatively, tension may also indicate remaining systematic biases in the measurements, which can have substantial impact on cosmological parameter measurements at the level of precision achieved by current data. Consequently, new physics in the neutrino sector is only a viable solution if the extra parameters eliminate the tension between data sets seen in the standard concordance cosmology, and is robustly confirmed by a variety of data sets. In this Letter, we show that sterile neutrinos do not relieve the tension between Planck and X-ray and SZ clusters, or with local measurements of $H_{0}$. Further, we show that the extended neutrino models are not preferred over the minimal model by any data combination, and that 
robust combinations of current measurements prefer low neutrino masses $\sum m_{\nu}, m_{\nu, \text { sterile }}^{\text {eff }} \lesssim 0.3 \mathrm{eV}$.

Data and methods.-We use CosmoMC [23] to constrain the parameters of the $\Lambda \mathrm{CDM}$ model extended with active $\left(+\mathrm{N}_{\text {eff }}, \sum m_{\nu}\right)$ and sterile $\left(+\mathrm{N}_{\text {eff }}, m_{\nu \text { sterile }}^{\text {eff }}\right)$ neutrinos, using combinations of the following data sets. CMB: the Planck CMB temperature likelihood [24], combined with Wilkinson Microwave Anisotropy Probe (WMAP) polarization [25], and high- $\ell$ temperature spectra from Atacama Cosmology Telescope and South Pole Telescope [26-28]. Lensing: the CMB lensing likelihood from Planck [29]. BAO: the Baryon Acoustic Oscillations (BAO) measurements from 6dF [30], Sloan Digital Sky Survey (SDSS) DR7 [31], WiggleZ [32], and Baryon Oscillation Spectroscopic Survey (BOSS) DR11 [33]. Shear: the weak lensing tomographic analysis from Canada-France Hawaii Telescope Lensing Survey (CFHTLenS) [34]. PlaSZ: the Planck SZ cluster abundances [2]. RSD: the RSD measurements from BOSS [13,35]. Xray: x-ray cluster mass function constraints [3]. HST: the $H_{0}$ measurement using supernovae by the Hubble Space Telescope [15]. Clustering: the three-dimensional galaxy power spectrum from WiggleZ [36,37], and the power spectrum of the reconstructed halo density field derived from Luminous Red Galaxies in SDSS DR7 [38], both up to $k=0.2 \mathrm{hMpc}^{-1}$. Note that we only use either the power spectrum or the BAO measurement from each data set.

Finally, we use the evidence ratio (or Bayes factor), which gives the relative odds of two models correctly describing the observations, under the assumption of equal a priori model probabilities (see, e.g., Refs. [21,39] and references therein). We calculate $\ln \left[E_{\Lambda \mathrm{CDM}} / E_{\mathrm{ext}}\right]$, the $\log$ arithm of the evidence ratio of the $\Lambda \mathrm{CDM}$ model divided by that of the extended neutrino models; thus, positive numbers favor the minimal model. In practice, since the models are nested, we compute evidence ratios with the Savage-Dickey density ratio, and we use kernel density estimation (KDE) to process Monte-Carlo Markov chains and reliably compute the marginalized posterior distributions at the $\Lambda \mathrm{CDM}$ values $\left(\sum m_{\nu}=0.06 \mathrm{eV}, m_{\nu, \text { sterile }}^{\text {eff }}=0.0 \mathrm{eV}\right.$,
$\mathrm{N}_{\text {eff }}=3.046$ ). The errors are calculated by jackknifing the KDE parameters. For all parameters, we consider the same prior ranges as the official Planck analysis [1]. However, the Bayes factors only depend on the neutrino parameters since we consider nested models. Specifically, we assume uniform priors in [0,5], [0, 3], and [3.046, 10] for $\sum m_{\nu}, m_{\nu, \text { sterile }}^{\text {eff }}$ and $\mathrm{N}_{\text {eff }}$, respectively, and we impose $m_{\nu, \text { sterile }}^{\text {eff }} /\left(\mathrm{N}_{\text {eff }}-3.046\right)<7 \mathrm{eV}$ to avoid a degeneracy between very massive neutrinos and cold dark matter.

No new concordance with sterile neutrinos. - Figure 1 shows constraints on the $\sigma_{8}-m_{\nu \text {,sterile }}^{\text {eff }}$ plane for several data combinations, including those used by Refs. [10-12]. Our minimal data set is $\mathrm{CMB}+\mathrm{BAO}$, since adding $\mathrm{BAO}$ to CMB does not shift the contours but constrains the matter density $\Omega_{m}$ and reduces the error bars (as expected for consistent data sets). However, the addition of the PlaSZ or Xray clusters, which prefer lower $\sigma_{8}$, shifts the contours significantly (by more than $2 \sigma$ ) outside the region allowed by $\mathrm{CMB}+\mathrm{BAO}$. This clearly indicates that the addition of sterile neutrinos to the $\Lambda \mathrm{CDM}$ model does not bring the $\mathrm{CMB}$ and cluster measurements into agreement. Note that the active scenario (not shown here) leads to similar results and tension, and does not yield concordance within the extended model either. Thus we may conclude that the tension must be resolved either by considering systematics in one or more of the relevant data sets, or else by new physics other than the introduction of massive (active or sterile) neutrinos. This is confirmed by the Bayes factor, presented in the first section of Table I, showing that the extended models are not preferred over the minimal $\Lambda \mathrm{CDM}$ model even in the presence of a tension.

Cluster cosmology is currently limited by modeling rather than statistical uncertainties [2]; thus, error bars on the x-ray, SZ, and optical clusters data used in Fig. 1 and in Refs. [10-14,16,17] may need to be significantly increased to account for additional potential systematics. The calibration of the mass-observable relation is critical for deriving robust cosmological constraints from clusters, and is complicated by uncertainties in mass measurements
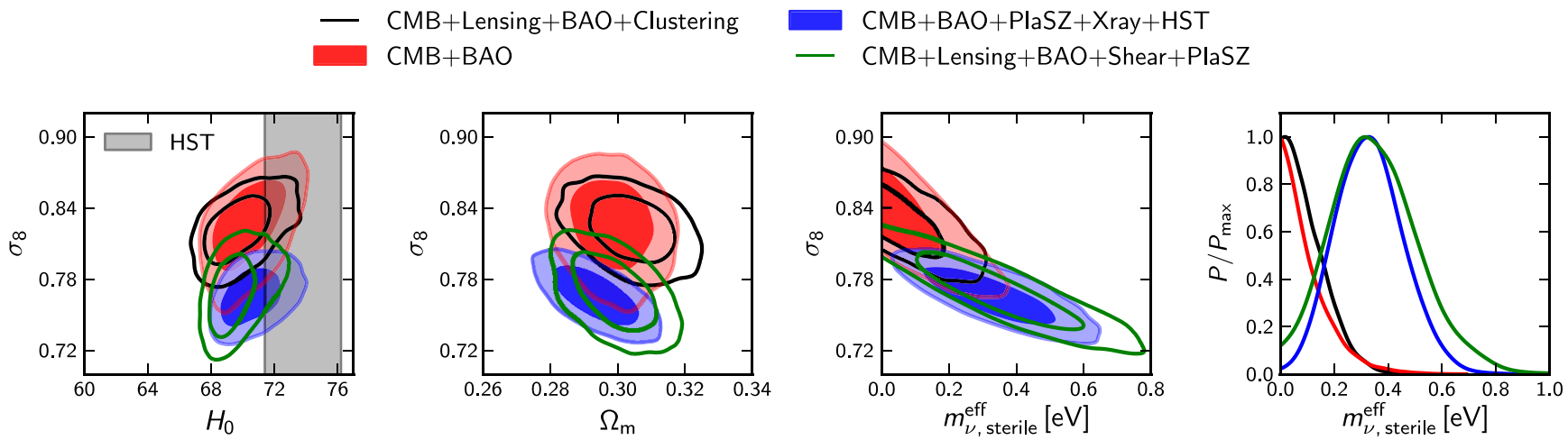

FIG. 1 (color online). Constraints on the $\Lambda \mathrm{CDM}+\mathrm{N}_{\mathrm{eff}}+m_{\nu, \text { sterile }}^{\text {eff }}$ model, showing that nonzero sterile neutrino mass is only favored as a result of a tension between the CMB and cluster data (PlaSZ, Xray) in the $\sigma_{8}-\Omega_{m}$ plane, and the degeneracy between $\sigma_{8}$ and neutrino mass. 
TABLE I. Evidence ratios $\ln \left[E_{\Lambda \mathrm{CDM}} / E_{\text {ext }}\right]$ between the minimal $\Lambda \mathrm{CDM}$ model and the extended neutrino models, in the active and sterile scenarios, showing that the extended models are not favored by any data combination. In particular, the upper part refers to the "tension" data combinations of Fig. 1, whereas the lower part corresponds to more robust data combinations (details in text), for which marginalized constraints are presented in Tables II and III.

\begin{tabular}{lcc}
\hline \hline & Active & Sterile \\
\hline CMB + BAO + PlaSZ + Xray + HST & $1.52_{-0.33}^{+0.16}$ & $-0.16_{-0.35}^{+0.39}$ \\
CMB + Lensing + BAO + Shear + PlaSZ & $3.77_{-0.09}^{+0.10}$ & $1.05_{-0.55}^{+0.26}$ \\
\hline $\mathrm{CMB}+\mathrm{BAO}$ & $4.42_{-0.05}^{+0.04}$ & $3.10_{-0.14}^{+0.07}$ \\
$\mathrm{CMB}+$ Lensing + BAO & $4.64_{-0.09}^{+0.03}$ & $2.99_{-0.05}^{+0.06}$ \\
$\mathrm{CMB}+$ Lensing + BAO + Clustering & $4.70_{-0.00}^{+0.02}$ & $3.35_{-0.13}^{+0.09}$ \\
$\mathrm{CMB}+$ Lensing + BAO + Clusters & $4.65_{-0.19}^{+0.10}$ & $2.61_{-0.23}^{+0.21}$ \\
$\mathrm{CMB}+$ Lensing + BAO + Shear & $4.32_{-0.16}^{+0.10}$ & $2.10_{-0.41}^{+0.21}$ \\
$\mathrm{CMB}+$ Lensing + BAO + RSD & $4.14_{-0.19}^{+0.10}$ & $1.81_{-0.09}^{+0.11}$ \\
\hline \hline
\end{tabular}

and the selection functions (see, e.g., Refs. [3,40]). Constraints on $\sigma_{8}$ from PlaSZ clusters are sensitive to assumptions and uncertainties in the modeling, as investigated in Ref. [2], and there are indications of a systematic mismatch between masses obtained via weak lensing compared with SZ masses [41]. The error bars on $\sigma_{8}\left(\Omega_{m}\right)^{\beta}$ from x-ray clusters used in Ref. [10] should be enlarged to account for confirmed sources of systematic uncertainties [3]. Interestingly, it was shown that the mass calibration by Ref. [42] from a self-consistent analysis of $\mathrm{x}$-ray, SZ, and optical scaling relations is consistent with a minimal flat $\Lambda \mathrm{CDM}$ model with no massive neutrinos $(1.7 \sigma)$, and is a better fit to additional data (e.g., $\left.H_{0}\right)$. Finally, the model dependence of these cluster constraints in the context of nonstandard models has not been investigated; therefore, it is unclear whether they can be used in a joint analysis in the context of such extended models.

If, after further investigation of such systematic effects, PlaSZ and Xray clusters remain in tension with $\mathrm{CMB}+\mathrm{BAO}$, this tension cannot be simply resolved by adding sterile neutrinos.

TABLE II. Marginalized 95\% C.L. constraints on the $\Lambda$ CDM + $\mathrm{N}_{\text {eff }}+\sum m_{\nu}$ model from a variety of robust LSS data sets with the Planck $\mathrm{CMB}$ temperature and lensing measurements. These data sets are not in tension and tightly constrain the mass of active neutrinos.

\begin{tabular}{lcc}
\hline \hline & $\sum m_{\nu}[\mathrm{eV}]$ & $\mathrm{N}_{\text {eff }}$ \\
\hline CMB + BAO & $<0.23$ & $<3.88$ \\
CMB + Lensing + BAO & $<0.25$ & $<3.84$ \\
CMB + Lensing + BAO + Clustering & $<0.26$ & $<3.80$ \\
CMB + Lensing + BAO + Clusters & $<0.29$ & $<3.78$ \\
CMB + Lensing + BAO + Shear & $<0.34$ & $<3.79$ \\
CMB + Lensing + BAO + RSD & $<0.37$ & $<3.75$ \\
\hline \hline
\end{tabular}

TABLE III. Same as Table II, but for the $\Lambda \mathrm{CDM}+\mathrm{N}_{\mathrm{eff}}+$ $m_{\nu, \text { sterile }}^{\text {eff }}$ model, showing tight constraints on the mass of sterile neutrinos.

\begin{tabular}{lcc}
\hline \hline & $m_{\nu \text {,sterile }}^{\text {eff }}[\mathrm{eV}]$ & $\mathrm{N}_{\text {eff }}$ \\
\hline CMB + BAO & $<0.28$ & $<3.91$ \\
CMB + Lensing + BAO & $<0.35$ & $<3.84$ \\
CMB + Lensing + BAO + Clustering & $<0.24$ & $<3.87$ \\
CMB + Lensing + BAO + Clusters & $<0.33$ & $<3.83$ \\
CMB + Lensing + BAO + Shear & $<0.51$ & $<3.82$ \\
CMB + Lensing + BAO + RSD & $<0.59$ & $<3.70$ \\
\hline \hline
\end{tabular}

Constraints on neutrino masses from robust data sets.We now investigate the constraints obtained on neutrino masses when combining data sets which are compatible and have been demonstrated to be robust to modeling uncertainties. Recent works using galaxy power spectra have obtained tight constraints on the mass of active neutrinos (e.g., Refs. [43-45]), and also showed that it could help in breaking degeneracies with the freedom in the primordial power spectrum from inflation [46]. For Clustering data, we use the power spectra from SDSS DR7 (reconstructed halo power spectrum) and WiggleZ (galaxy power spectrum), truncated at $k=0.2 \mathrm{~h} \mathrm{Mpc}^{-1}$ in order to avoid nonlinear scales, marginalizing over the galaxy bias. For Shear data, we use the tomographic weak gravitational lensing analysis by the CFHTLenS [34], which were shown to be usable in neutrino extensions of $\Lambda \mathrm{CDM}$ [13]. For the Clusters data, we use the thermal SZ measurements from cross correlation of the CMB with $\mathrm{x}$-ray clusters [47], which are the most recent cluster-derived cosmological constraints. They rely on cross correlations and were also demonstrated to be robust to the choices in the modeling and data (tested with Planck and WMAP). We jointly use the Planck CMB temperature and Lensing power spectra (to probe the growth of structure with the $\mathrm{CMB}$ ) with the $B A O$ constraints (to constrain $\Omega_{m}$ ). Finally, we also use the RSD measurements from BOSS [35].

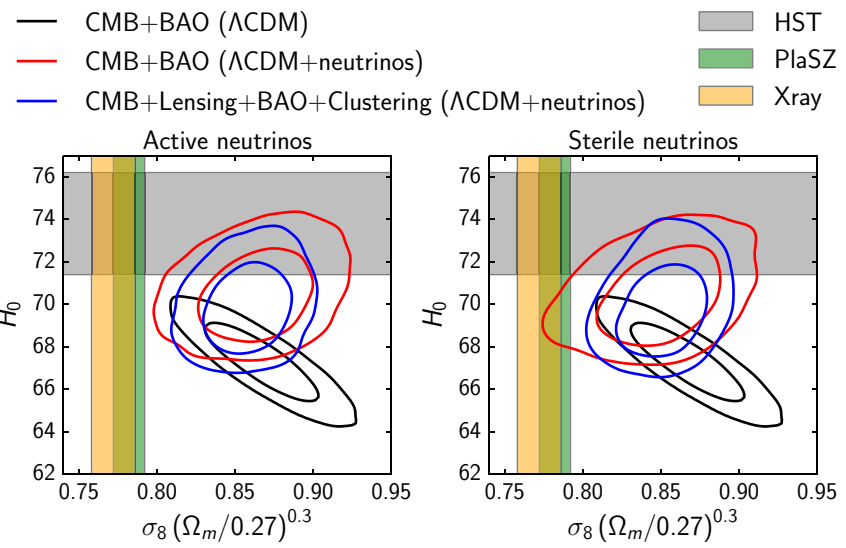

FIG. 2 (color online). Persistence of the tension as the minimal $\Lambda \mathrm{CDM}$ model is extended in the neutrino sector, i.e., as $\mathrm{N}_{\text {eff }}$ and massive active or sterile neutrinos are added. 
- $\mathrm{CMB}+$ Lensing $+\mathrm{BAO}+$ Clustering

- $\mathrm{CMB}+\mathrm{BAO}+\mathrm{Xray}+\mathrm{HST}$

$\mathrm{CMB}+\mathrm{BAO}+$ Xray $+\mathrm{HST}+\mathrm{BICEP}$
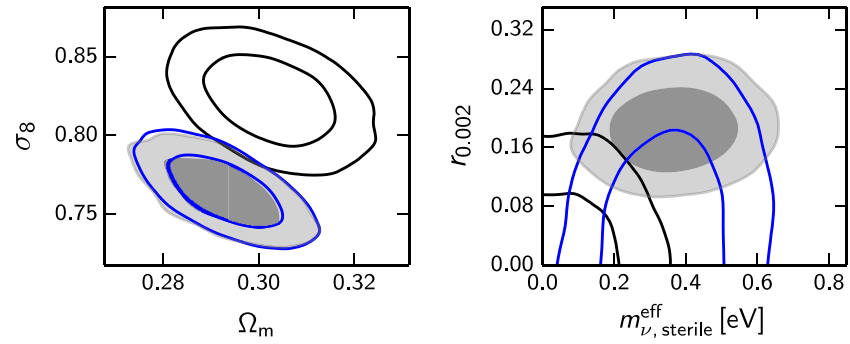

FIG. 3 (color online). Constraints on the $\Lambda \mathrm{CDM}+r_{0.002}+\mathrm{N}_{\text {eff }}+$ $m_{\nu, \text { sterile }}^{\text {eff }}$ model, illustrating the persisting tension between $\mathrm{x}$-ray clusters and $\mathrm{CMB}+\mathrm{BAO}$ in the $\sigma_{8}-\Omega_{m}$ plane, despite an apparent reconciliation of the BICEP and Planck results on $r_{0.002}$.

Tables II and III summarize the constraints on neutrino masses in the active and sterile neutrino scenarios, respectively, i.e., $\Lambda \mathrm{CDM}+\mathrm{N}_{\mathrm{eff}}+m_{\nu, \text { sterile }}^{\text {eff }}$ and $\Lambda \mathrm{CDM}+\mathrm{N}_{\mathrm{eff}}+$ $m_{\nu, \text { sterile }}^{\text {eff }}$ models, arising from a variety of data combinations. We see that multiple combinations yield similar constraints, and tend to small neutrino masses, e.g., $\sum m_{\nu}$, $m_{\nu, \text { sterile }}^{\text {eff }} \lesssim 0.3 \mathrm{eV}$ at $95 \%$ C.L. Note that some of these constraints may be relaxed by adding freedom to the model, for example to the primordial power spectrum [46]. Interestingly, as also noted by Ref. [13], the Shear and RSD data prefer lower $\sigma_{8}$ and, thus, larger neutrino mass. However, the Bayes factors presented in the second section of Table I indicate a preference for the minimal $\Lambda \mathrm{CDM}$ model in all cases, even with the Shear and RSD data. Note that Ref. [13] marginalized over the lensing information which, as is well known [1], leads to a preference for higher $\sigma_{8}$; conversely, our analysis combined the CMB temperature and lensing information.

Figure 2 illustrates the persistence of the tension between the $\mathrm{CMB}+\mathrm{BAO}$, HST, PlaSZ, and Xray data, as one extends the minimal $\Lambda \mathrm{CDM}$ model in the neutrino sector. The tension with local measurements of $H_{0}$ is alleviated by $\mathrm{N}_{\text {eff }}$ because of the degeneracy between these parameters $[19,21]$, but the tension with PlaSZ and Xray clusters persists despite the addition of both $\mathrm{N}_{\mathrm{eff}}$ and neutrino masses. The levels of tension are comparable in minimal and extended models when adding Lensing and Clustering data. We note that the PlaSZ and Xray constraints were derived for the $\Lambda \mathrm{CDM}$ model, and it is unclear whether they can be used in the context of the extended models. In contrast, the data sets used in Tables II and III all relied on uncompressed likelihoods or constraints shown to be usable within the extended models.

Finally, sterile neutrinos were claimed $[16,17]$ to also resolve the tension in the Planck measurements of the tensor-to-scalar ratio $\left(r_{0.002}<0.11\right.$ at $95 \%$ C.L. $)$ and the recent BICEP result, $r_{0.002}=0.2_{-0.05}^{+0.07}$ [18]. However, the tension in the $\sigma_{8}-\Omega_{m}$ plane detailed previously persists
TABLE IV. Evidence ratios $\ln \left[E_{\Lambda \mathrm{CDM}} / E_{\mathrm{ext}}\right]$ between the minimal $\Lambda$ CDM model and the $\Lambda \mathrm{CDM}+r_{0.002}+\mathrm{N}_{\text {eff }}+m_{\nu, \text { sterile }}^{\text {eff }}$ model, showing that sterile neutrinos are not favored by the data, even when adding the BICEP results.

\begin{tabular}{lc}
\hline \hline & Sterile \\
\hline CMB + Lensing + BAO + Clustering & $2.89_{-0.19}^{+0.13}$ \\
CMB + BAO + Xray + HST & $-0.70_{-0.02}^{+0.07}$ \\
CMB + BAO + Xray + HST + BICEP & $-0.66_{-0.04}^{+0.05}$ \\
\hline \hline
\end{tabular}

in the extended model $\Lambda \mathrm{CDM}+r_{0.002}+\mathrm{N}_{\text {eff }}+m_{\nu, \text { sterile }}^{\text {eff }}$, as shown in Fig. 3. Hence, the relaxed constraints on $r_{0.002}$ from this data combination originates from a compromise between data sets in tension, not a new concordance. This is confirmed by the Bayes factors, presented in Table IV, showing that the extended model is not favored over $\Lambda$ CDM.

Conclusions.-The need for extra parameters yielding a new cosmological concordance can only be convincing if the combined data sets are in tension in the minimal model, and in agreement in extended model. We show that massive sterile neutrinos do not bring about a new cosmic concordance, but rather highlight the tension between the $\mathrm{CMB}+$ $\mathrm{BAO}$ and SZ or X-ray clusters. A compilation of current LSS data which have been demonstrated to be robust to modeling uncertainties, when combined with Planck, tend to small masses $\sum m_{\nu}, m_{\nu, \text { sterile }}^{\text {eff }} \lesssim 0.3 \mathrm{eV}$ at $95 \%$ C.L. in the context of the $\Lambda \mathrm{CDM}$ model extended with $\mathrm{N}_{\mathrm{eff}}$ and neutrino mass parameters. Similarly, as found in Refs. $[19,21]$ the data cannot distinguish between $\mathrm{N}_{\text {eff }} \sim 3$ and 4 and do not favor extra neutrinos over the standard three families. These conclusions are corroborated by the Bayesian evidence: the more complex models are not preferred, even when using data sets in tension. We conclude that current cosmological constraints do not provide evidence for large neutrino masses or extra neutrinos, even in the presence of the tension between Planck CMB and SZ and x-ray clusters. If this tension does not resolve after further investigation of systematic effects, new physics beyond massive neutrinos will be necessary to reconcile these data sets.

We thank Ofer Lahav, Stephen Feeney, Nina Roth, Florian Beutler, August Evrard, Raphael Flauger, Marta Spinelli, and Filipe Abdalla for useful discussions. B. L. is supported by the Perren Fund and the IMPACT Fund. H. V. P. is supported by STFC and the European Research Council under the European Community's Seventh Framework Programme (FP7/2007-2013)/ERC Grant Agreement No. 306478CosmicDawn. L. V. is supported by the European Research Council under the European Community's Seventh Framework Programme FP7-IDEAS-Phys.LSS 240117 and Mineco Grant No. FPA2011-29678- C02-02. Based on observations obtained with Planck [48], a ESA science mission with instruments and contributions directly funded by ESA member states, NASA, and Canada. 
*boris.leistedt.11@ucl.ac.uk

h.peiris@ucl.ac.uk

†iciaverde@icc.ub.edu

[1] P. A. R. Ade, N. Aghanim, C. Armitage-Caplan, M. Arnaud, M. Ashdown, F. Atrio-Barandela, J. Aumont, C. Baccigalupi, A. J. Banday et al. (Planck Collaboration), arXiv:1303.5076.

[2] P. A. R. Ade, N. Aghanim, C. Armitage-Caplan, M. Arnaud, M. Ashdown, F. Atrio-Barandela, J. Aumont, C. Baccigalupi, A. J. Banday et al. (Planck Collaboration), arXiv:1303.5080.

[3] A. Vikhlinin, A. V. Kravtsov, R. A. Burenin, H. Ebeling, W. R. Forman, A. Hornstrup, C. Jones, S. S. Murray, D. Nagai, H. Quintana et al., Astrophys. J. 692, 1060 (2009).

[4] J. Beringer, J. F. Arguin, R. M. Barnett, K. Copic, O. Dahl, D. E. Groom, C. J. Lin, J. Lys, H. Murayama, C. G. Wohl et al. (Particle Data Group), Phys. Rev. D 86, 010001 (2012).

[5] M. Gonzalez-Garcia, M. Maltoni, J. Salvado, and T. Schwetz, J. High Energy Phys. 12 (2012) 123.

[6] J. M. Conrad, W. C. Louis, and M. H. Shaevitz, Annu. Rev. Nucl. Part. Sci. 63, 45 (2013).

[7] K. Abazajian, M. Acero, S. Agarwalla, A. Aguilar-Arevalo, C. Albright et al., arXiv:1204.5379.

[8] J. Lesgourgues and S. Pastor, Phys. Rep. 429, 307 (2006).

[9] L. Verde, P. Protopapas, and R. Jimenez, Phys. Dark Univ. 2, 166 (2013).

[10] R. A. Battye and A. Moss, Phys. Rev. Lett. 112, 051303 (2014).

[11] M. Wyman, D. H. Rudd, R. A. Vanderveld, and W. Hu, Phys. Rev. Lett. 112, 051302 (2014).

[12] J. Hamann and J. Hasenkamp, J. Cosmol. Astropart. Phys. 10 (2013) 044.

[13] F. Beutler, S. Saito, J. R. Brownstein, C.-H. Chuang, A. J. Cuesta, W. J. Percival, A. J. Ross, N. P. Ross, D. P. Schneider, L. Samushia et al., arXiv:1403.4599.

[14] E. Giusarma, E. Di Valentino, M. Lattanzi, A. Melchiorri, and O. Mena, arXiv:1403.4852.

[15] A. G. Riess, L. Macri, S. Casertano, H. Lampeitl, H. C. Ferguson, A. V. Filippenko, S. W. Jha, W. Li, and R. Chornock, Astrophys. J. 730, 119 (2011); , 732, 129(E) (2011).

[16] J.-F. Zhang, Y.-H. Li, and X. Zhang, arXiv:1403.7028.

[17] C. Dvorkin, M. Wyman, D. H. Rudd, and W. Hu, arXiv:1403.8049.

[18] P. A. R. Ade, R. W. Aikin, D. Barkats, S. J. Benton, C. A. Bischoff, J. J. Bock, J. A. Brevik, I. Buder, E. Bullock et al. (BICEP2 Collaboration), Phys. Rev. Lett. 112, 241101 (2014).

[19] S. M. Feeney, H. V. Peiris, and L. Verde, J. Cosmol. Astropart. Phys. 04 (2013) 036.

[20] J. Hu, R. Cai, Z. Guo, and B. Hu, J. Cosmol. Astropart. Phys. 05 (2014) 020.

[21] L. Verde, S. M. Feeney, D. J. Mortlock, and H. V. Peiris, J. Cosmol. Astropart. Phys. 09 (2013) 013.

[22] G. Efstathiou, arXiv:1311.3461.

[23] A. Lewis and S. Bridle, Phys. Rev. D 66, 103511 (2002).

[24] P. A. R. Ade, N. Aghanim, C. Armitage-Caplan, M. Arnaud, M. Ashdown, F. Atrio-Barandela, J. Aumont, C. Baccigalupi, A. J. Banday et al. (Planck collaboration), arXiv:1303.5075.
[25] C. Bennett, D. Larson, J. Weiland, N. Jarosik, G. Hinshaw et al., Astrophys. J. Suppl. Ser. 208, 20 (2013).

[26] R. Keisler, C. L. Reichardt, K. A. Aird, B. A. Benson, L. E. Bleem, J. E. Carlstrom, C. L. Chang, H. M. Cho, T. M. Crawford, A. T. Crites et al., Astrophys. J. 743, 28 (2011).

[27] S. Das, T. Louis, M. R. Nolta, G. E. Addison, E. S. Battistelli, J. Bond, E. Calabrese, D. C. M. J. Devlin, S. Dicker, J. Dunkley et al., J. Cosmol. Astropart. Phys. 04 (2014) 014.

[28] C. L. Reichardt, L. Shaw, O. Zahn, K. A. Aird, B. A. Benson, L. E. Bleem, J. E. Carlstrom, C. L. Chang, H. M. Cho, T. M. Crawford et al., Astrophys. J. 755, 70 (2012).

[29] P. A. R. Ade, N. Aghanim, C. Armitage-Caplan, M. Arnaud, M. Ashdown, F. Atrio-Barandela, J. Aumont, C. Baccigalupi, A. J. Banday et al. (Planck Collaboration), arXiv:1303.5077.

[30] F. Beutler, C. Blake, M. Colless, D. H. Jones, L. StaveleySmith, L. Campbell, Q. Parker, W. Saunders, and F. Watson, Mon. Not. R. Astron. Soc. 416, 3017 (2011).

[31] N. Padmanabhan, X. Xu, D. J. Eisenstein, R. Scalzo, A. J. Cuesta, K. T. Mehta, and Eyal Kazin, Mon. Not. R. Astron. Soc. 427, 2132 (2012).

[32] C. Blake, E. Kazin, F. Beutler, T. Davis, D. Parkinson et al., Mon. Not. R. Astron. Soc. 418, 1707 (2011).

[33] L. Anderson, E. Aubourg, S. Bailey, F. Beutler, V. Bhardwaj, M. Blanton, A. S. Bolton, J. Brinkmann, J. R. Brownstein, A. Burden et al., arXiv:1312.4877.

[34] M. Kilbinger, L. Fu, C. Heymans, F. Simpson, J. Benjamin et al., Mon. Not. R. Astron. Soc. 430, 2200 (2013).

[35] F. Beutler, S. Saito, H.-J. Seo, J. Brinkmann, K. S. Dawson, D. J. Eisenstein, A. Font-Ribera, S. Ho, C. K. McBride, F. Montesano et al., arXiv:1312.4611.

[36] C. Blake, S. Brough, M. Colless, W. Couch, S. Croom et al., Mon. Not. R. Astron. Soc. 406, 803 (2010).

[37] D. Parkinson, S. Riemer-Sorensen, C. Blake, G. B. Poole, T. M. Davis et al., Phys. Rev. D 86, 103518 (2012).

[38] B. A. Reid et al., Mon. Not. R. Astron. Soc. 404, 60 (2010).

[39] R. T. Cox, American Journal of physical anthropology Supplement: the official publication of the American Association of Physical Anthropologists 14, 1 (1946).

[40] E. Rozo, E. S. Rykoff, J. G. Bartlett, and A. E. Evrard, arXiv:1302.5086.

[41] A. von der Linden, A. Mantz, S. W. Allen, D. E. Applegate, P. L. Kelly, R. G. Morris, A. Wright, M. T. Allen, P. R. Burchat, D. L. Burke et al., arXiv:1402.2670.

[42] E. Rozo, J. G. Bartlett, A. E. Evrard, and E. S. Rykoff, Mon. Not. R. Astron. Soc. 438, 78 (2014).

[43] S. A. Thomas, F. B. Abdalla, and O. Lahav, Phys. Rev. Lett. 105, 031301 (2010).

[44] E. Giusarma, R. de Putter, S. Ho, and O. Mena, Phys. Rev. D 88, 063515 (2013).

[45] S. Riemer-Sorensen, D. Parkinson, and T. M. Davis, arXiv:1306.4153.

[46] R. de Putter, E. V. Linder, and A. Mishra, Phys. Rev. D 89, 103502 (2014)

[47] A. Hajian, N. Battaglia, D. N. Spergel, J. R. Bond, C. Pfrommer, and J. L. Sievers, J. Cosmol. Astropart. Phys. 11 (2013) 064.

[48] http://www.esa.int/Planck. 